\title{
Culturally informed research design issues in a study for MDMA-assisted psychotherapy for posttraumatic stress disorder
}

\author{
MONNICA T. WILLIAMS ${ }^{1,2 *}$, SARA REED ${ }^{1}$ and RITIKA AGGARWAL ${ }^{3}$ \\ ${ }^{1}$ Department of Psychology, University of Connecticut, Storrs, CT, USA \\ ${ }^{2}$ School of Psychology, University of Ottawa, Ottawa, ON, Canada \\ ${ }^{3}$ MAPS Public Benefit Corporation, Santa Cruz, CA, USA
}

(Received: May 1, 2019; accepted: June 13, 2019)

\begin{abstract}
Recent research suggests that psychedelic drugs can be powerful agents of change when utilized in conjunction with psychotherapy. Methylenedioxymethamphetamine (MDMA)-assisted psychotherapy has been studied as a means of helping people overcome posttraumatic stress disorder, believed to work by reducing fear of traumatic memories and increasing feelings of trust and compassion toward others, without inhibiting access to difficult emotions. However, research studies for psychedelic psychotherapies have largely excluded people of color, leaving important questions unaddressed for these populations. At the University of Connecticut, we participated as a study site in a MAPSsponsored, FDA-reviewed Phase 2 open-label multisite study, with a focus on providing culturally informed care to people of color. We discuss the development of a study site focused on the ethnic minority trauma experience, including assessment of racial trauma, design of informed consent documents to improve understanding and acceptability to people of color, diversification of the treatment team, ongoing training for team members, validation of participant experiences of racial oppression at a cultural and individual level, examination of the setting and music used during sessions for cultural congruence, training for the independent rater pool, community outreach, and institutional resistance. We also discuss next steps in ensuring that access to culturally informed care is prioritized as MDMA and other psychedelics move into late phase trials, including the importance of diverse sites and training focused on therapy providers of color.
\end{abstract}

Keywords: MDMA, race, methodology, PTSD, racial trauma, psychedelic

\section{INTRODUCTION}

Posttraumatic stress disorder (PTSD) describes a constellation of symptoms that can arise after experiencing or witnessing a traumatic event. Individuals who have been exposed to a traumatic event that causes a serious fear for their own life or the lives of others is at risk of developing PTSD, with those experiencing multiple traumas at higher risk (Breslau, Chilcoat, Kessler, \& Davis, 1999). People typically affected include survivors of violent acts and disasters, emergency responders to traumatic events, people who experience the sudden death of a loved one, victims of abuse, accident victims, and combat veterans (Kessler et al., 2017).

However, many other events can be traumatic as well, particularly to people of color (Williams, Printz, Ching, \& Wetterneck, 2018). African Americans, Hispanic/Latinx Americans, and Native Americans are more likely to experience trauma related to experiences of police brutality, community violence, racism, and poverty than their nonHispanic White counterparts (Pole, Gone, \& Kulkarni, 2008; Williams, Printz, et al., 2018). Many immigrants and refugees have PTSD from the impact of cultural trauma due to ethnic cleansing and war, compounded by difficult immigration experiences and adjustment-related stressors (Bosson et al., 2017). Therefore, addressing the problem of traumatization among these often marginalized ethnic groups is an important goal for an equitable and healthy society.

The Multidisciplinary Association for Psychedelic Studies (MAPS) and MAPS Public Benefit Corporation (MAPS PBC) have been at the forefront of addressing PTSD by working to make 3,4-methylenedioxymethamphetamine (MDMA)-assisted psychotherapy into a legal prescription medicine (Emerson, Ponté, Jerome, \& Doblin, 2014). MAPS is currently sponsoring Phase 3 clinical trials of MDMA as a tool to assist psychotherapy for severe PTSD, with clinical sites in the United States, Canada, and Israel. MDMA is also known as "ecstasy" or "molly," but those street drugs frequently also contain unknown and/or dangerous adulterants. In MDMA-assisted psychotherapy, pure MDMA is administered only 2-3 times in conjunction with psychotherapy, unlike most medications for mental illnesses, which are often taken daily for several years (e.g., Mithoefer, Wagner, Mithoefer, Jerome, \& Doblin, 2011).

Studies suggest that MDMA can augment psychotherapeutic work to help improve symptoms of PTSD

\footnotetext{
* Corresponding author: Monnica T. Williams, PhD; School of Psychology, University of Ottawa, 136 Jean-Jacques Lussier, Vanier Hall, Ottawa, ON K1N 6N5, Canada; Phone: +1 613 562 5801; Fax: +1 613562 5169; E-mail: mwilli25@uottawa.ca
}

This is an open-access article distributed under the terms of the Creative Commons Attribution-NonCommercial 4.0 International License, which permits unrestricted use, distribution, and reproduction in any medium for non-commercial purposes, provided the original author and source are credited, a link to the CC License is provided, and changes - if any - are indicated. 
(e.g., Mithoefer et al., 2018). MDMA reduces the fear of traumatic memories and increases feelings of trust and compassion toward others without causing sensory distortions or inhibiting access to difficult emotions. As such, MDMA can increase the effectiveness of psychotherapy by making traumatic memories more accessible and strengthening the alliance between therapist and client, making it an important and promising new intervention for the treatment of PTSD. In August 2017, the US Food and Drug Administration (FDA) granted "Breakthrough Therapy Designation" to MDMA-assisted psychotherapy for the treatment of PTSD. This indicates that the FDA believes, based on Phase 2 data, that MDMA-assisted psychotherapy may have an advantage over existing medications for PTSD, and it signals their intent to expedite the review process for a New Drug Application for MDMA (Burns, 2017).

However, there are still large gaps in the body of knowledge surrounding MDMA. Although many communities of color experience the highest rates of PTSD - leading to prolonged suffering, disability, and poor quality of life (e.g., Himle, Baser, Taylor, Campbell, \& Jackson, 2009) they are extremely underrepresented in psychedelic research (Michaels, Purdon, Collins, \& Williams, 2018; Williams \& Leins, 2016). People of color have not been adequately included in previous studies of MDMA-assisted psychotherapy to determine if the treatment is appropriate, safe, or effective for them. Research has been conducted mostly with White participant samples, limiting the generalizability of safety and efficacy findings for people of color, and leading to their exclusion from MDMA's potential therapeutic benefits (e.g., Herzberg \& Butler, 2019). Thus, lack of diversity is an important ethical concern (Sisti, 2018).

\section{CULTURALLY INFORMED ADAPTATIONS TO MDMA-ASSISTED PSYCHOTHERAPY}

At the University of Connecticut Health Center (UConn), our team participated in a MAPS-sponsored, Phase 2 openlabel multisite study for PTSD (MP-16), under the guidance of the FDA. This site was the only one focused on the recruitment of ethnoracial minority participants meeting criteria for PTSD. The site protocol emphasized culturally sensitive and respectful treatment approaches for people of diverse backgrounds, considering the impact of cultural differences and the possible need to adapt MDMA-assisted psychotherapy to the needs and culturally specific traumas experienced by people of color. In this study, we discuss some of methodological changes that were made to help ensure that the MDMA research was culturally sensitive and inclusive.

\section{Diversification of the treatment team}

Several studies have documented to have adequate staff members from diverse groups as key to successful efforts at recruitment and retention of people of color (Williams, Tellawi, Wetterneck, \& Chapman, 2013). One reason for this is that many people of color fear or feel uncomfortable with the mental health system (U.S. Department of Health and Human Services, 1999). Anecdotal reports from people of color reveal fear and uncertainty from White therapists over the ability to support the complexity of their emotional experience, and indeed, several potential participants expressed gratitude, safety, and comfort when interacting with our therapists of color, as they had experienced disconnects with White clinicians in the past. It can make a critical difference in participation, willingness, and overall participant comfort when members of the research team represent participant diversity. It makes the outcome of the study more personally relevant when participants see others like themselves invested in the research program and occupying key leadership roles (e.g., Williams, Proetto, Casiano, \& Franklin, 2012), and participants may feel less worried about experiencing racism when they see other people of color involved. Furthermore, having clinicians from different ethnic groups improves the ability of the team as a whole to understand and appreciate issues relevant to a greater number of potential participants.

At UConn, we intentionally made our research team as diverse as possible. This included the Principal Investigator (PI; an African American female psychologist), the co-PI (a White American male psychiatrist), the study coordinator (an African American female marriage and family therapist), the study physician (an Indian American male), and three additional study therapists that included a Singaporean Chinese male, biracial African American female, and a Hispanic Asian American male. Our team was also diverse in terms of sexual identity, age, religious beliefs, clinical training, and therapeutic orientation. By sharing each other's experience and perspectives, this diversity enabled us to effectively work with a range of clients with several different ethnic and intersecting identities.

\section{Outreach and recruitment}

Most of the sites in the MAPS PBC MDMA-assisted psychotherapy studies are contacted directly by prospective participants as a result of information posted on the clinical trials registry (clinicaltrials.gov) and e-mail announcements from MAPS. However, these potential participants tend to have a preexisting interest in the study due to something they saw in the media or a previous connection with psychedelic communities, which are, with few exceptions, mostly White. As a result, the information about the study was not being adequately disseminated to communities of color.

Although MAPS PBC, the designated clinical trial organizer, had preapproved language for recruitment materials, as shown in Figure 1, it was inadequate for attracting people of color. Unlike the White participants who were flooding the study lines at all sites, our target audience required a different approach, which included building trust, psychoeducation, and gentle encouragement. The approved text did not address the concerns of our participants of color, such as fears of research abuse and safety of the drug. Due to our country's long history of research abuses against people of color (Suite, La Bril, Primm, \& Harrison-Ross, 2007), minimal information could make potential participants believe that study sponsors were hiding things from them 


\section{Do You Have Posttraumatic Stress Disorder (PTSD)?}

\section{We are seeking volunteers for a research study who are at least 18 years old and have been diagnosed} with PTSD. You must be in good physical health to participate. We are studying an investigational drug (MDMA) used in combination with psychotherapy. The study takes place in [Study Site Location].

For more information, please call [site phone number] or email [study coordinator] or see maps.org/research/mdma.

Figure 1. Original IRB-approved ad for MDMA-assisted psychotherapy study for PTSD

and generate increased suspicion about the researchers' intentions.

MAPS PBC was not amenable to investing time and funds into creating new ads that would attract people of color when the existing methods seemed to be generating more than enough participants. To fill this gap, for the next phase of the study, our site created its own flyer to target these underrepresented groups, based on the literature and our own past experience with minority outreach efforts. As shown in Figure 2, the new ad included several vital elements including (a) names and pictures of study staff; (b) diverse and well-qualified team leaders; (c) psychoeducation about PTSD and common causes of trauma among people of color; (d) language addressing fears of death, disability, and addiction; and (e) language about our site's culturally sensitive and respectful treatment approach (Avery, Hernandez, \& Hebl, 2004; Williams, Proetto, et al., 2012). Such ads can be used for campus posters, public transportation, handouts, web pages, and e-mail outreach.

However, ads alone are not usually sufficient to generate adequate interest in a research study, particularly a potentially controversial one that included a psychedelic drug. The literature and our own experience indicated that word of mouth from trusted sources is one of the most powerful recruiting tools for research studies (Williams, BeckmannMendez, \& Turkheimer, 2013). As such, community outreach is an important part of a successful recruitment effort, and study personnel can help by making and maintaining personal connections with important people and organizations within communities of interest. At UConn, our team gave several talks both on and off campus. On campus, lectures were co-sponsored by Students for Sensible Drug Policy who partnered with Collective Uplift, an organization for student athletes of color. The topics of the talks were intended to be engaging and relevant to students in these organizations; lectures included "From Microaggressions to Hate Crimes: \#RacialTraumaIsReal," given by the PI, and "MDMA-Assisted Psychotherapy: Our Experiences as Subjects in an FDA-Approved Clinical Trial," given by two of the study therapists who participated in a healthy volunteer study sponsored by MAPS (e.g., Ching, in press). Each of these talks generated a number of interested potential participants of color from those who had attended the event as well as interest from their friends and families. When giving such talks, study personnel can provide flyers for people to take home and share, such as those in Figure 2.

It should be noted that some of the unsolicited e-mails we received from participants were from people of color, but there was no way to know this since most participants did not disclose their race or ethnicity in their initial e-mails to us. One strategy that helped was to include an auto-reply e-mail that acknowledged receipt of the potential participant's e-mail and asked them to respond by e-mail with specific demographic information, including their race/ ethnicity, as well as other demographics such as age, city of residence, and current medications. This strategy is currently in use at several of the Phase 3 sites to more efficiently identify racially and ethnically diverse participants.

\section{Compensation}

People of color are more likely to be economically disadvantaged, making it more difficult to participate, as the time spent in a research study may be outweighed by other responsibilities, such as a job, school, or taking care of a family (Fisher et al., 2002). Therefore, it is important to provide meaningful and adequate compensation to participants for their time and to show appreciation for their participation (Williams, Tellawi, et al., 2013). Furthermore, friends and family members may be suspicious of research, or in the case of mental health specifically, consider it a luxury, and pressure participants of color not to participate. Incentives can help persuade others in the participant's circle that the participant's time is being respected and the study is worth conducting. Although there may be concerns about increased study costs, the funds that go toward compensating participants are actually a very small percentage of the study budget overall, and such incentives facilitate participation of low-income and economically disadvantaged individuals of all racial groups.

Because of the great interest by White participants, initially there did not appear to be a need to financially compensate participants. However, we believe that all participants should be fairly compensated as a matter of social justice. That being said, payments that are too large can amount to coercion and thus the appropriate amount should be carefully considered (Grady, 2005). At certain approved sites, MAPS was able to provide a total of $\$ 400$ to 


\section{Do you have Posttraumatic Stress Disorder (PTSD)?}

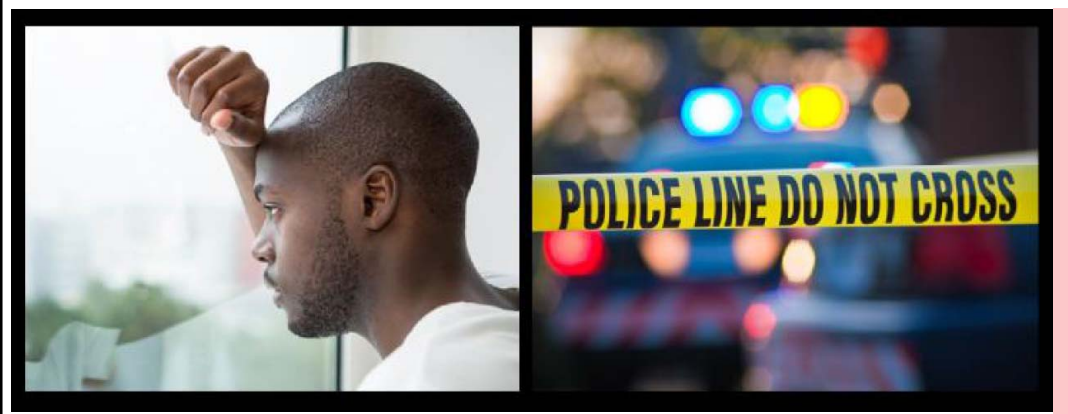

\section{Who Gets PTSD?}

PTSD can be caused by experiencing or witnessing extremely upsetting or life-threatening events. PTSD can affect people of any age, race, gender, or ethnicity, however people of color seek treatment less often than Whites.

\section{New PTSD Therapy Study at UConn}

We are expanding an FDA-approved research study to the University of Connecticut Health Center to determine whether the drug MDMA combined with psychotherapy is helpful for people who have PTSD.

MDMA-assisted psychotherapy seems to be helpful for PTSD, but so far people of color have not been included in meaningful numbers. Our work is focused on culturally-sensitive and respectful treatment approaches for people from diverse backgrounds.

To date, over 1,284 people have been given MDMA in controlled research settings. There have been no deaths, disabilities, or addictions as a result, and no serious unexpected medical issues happened. In this study, MDMA is administered a few times over 12 weeks, unlike most medications for PTSD which are often taken daily for years.

For more information, call (860) 679-2631, email ptsdstudy@uconn.edu, or visit maps.org/research/mdma. \#RacialTraumalsReal www.mentalhealthdisparities.org

People of Color May Have Trauma Due to:

- Racism and Discrimination

- Workplace Threats or Harassment

- Racial Profiling

- Assault by Law Enforcement

- Urban or Gang Violence

- Incarceration

- Pregnancy \& Childbirth Experiences

Immigrants and Refugees

May Have Traumas Due to:

- Ethnic Cleansing \& Persecution

- Experiencing or Witnessing Torture

- Living in a War Zone

- Immigration Difficulties

- Deportation

Other Common Sources of Trauma:

- Military Service

- Motor Vehicle Accidents

- Fire or Natural Disasters

- Domestic Violence

- Physical or Sexual Abuse

- Medical Conditions \& Procedures

Some Symptoms of PTSD:

- Feeling the World is Unsafe

- Upsetting Memories

- Anger and Irritability

- Loss of Trust in Others

- Feeling Emotionally Numb

- Feeling Your Life Will be Cut Short

- Avoiding Upsetting Reminders of Trauma

- Often Feeling on High-Alert

- Depression and Sadness

- Difficulty Sleeping or Nightmares

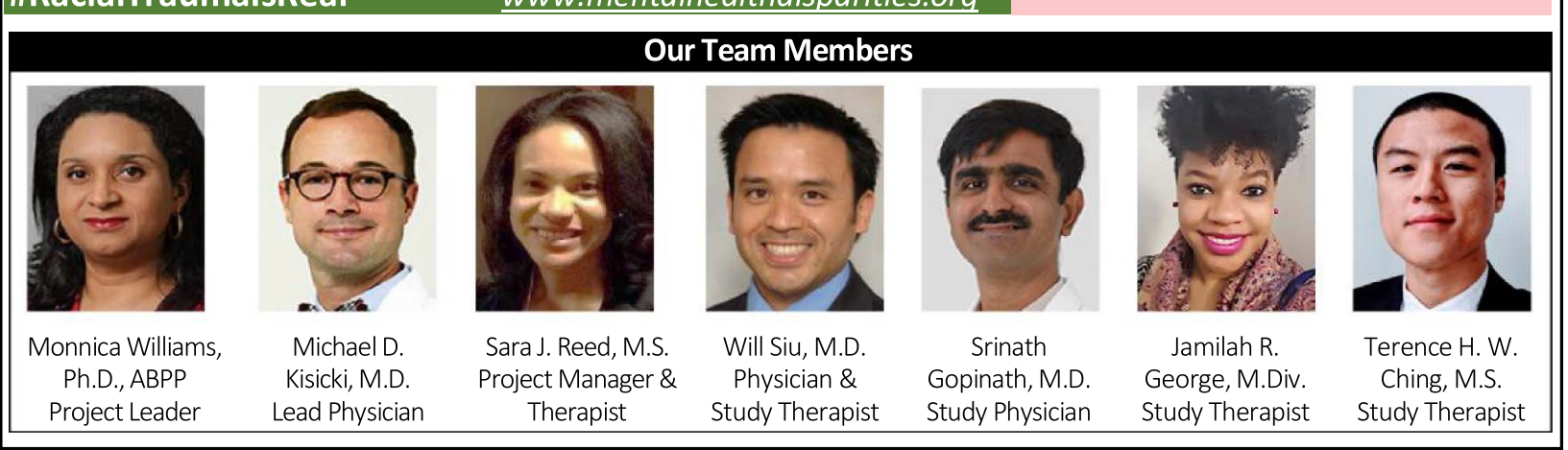

Figure 2. Sample recruitment flyer. A colorful flyer, originally designed for future Phase 3 studies, illustrates important design elements that are key for attracting people of color

participants $-\$ 100$ for each overnight visit and $\$ 100$ for the independent rater assessments (This is explained in more detail in the section "Training for independent raters."). This greatly facilitated participation as several of our participants were low income or students. Compensation should be provided in cash whenever possible, as a check can be a dilemma if participants do not have checking accounts, which was the case for some of our participants. 


\section{Initial screening}

For the initial screening, clinicians need to obtain detailed information from potential participants by phone with very little time to build rapport. Given that many participants of color are mistrustful of the medical system, and even more so a medical research study, extra effort needs to occur to create trust. Often people of color have not felt respected or been given the same courtesy that most of the White participants have taken for granted in medical contexts (Maina, Belton, Ginzberg, Singh, \& Johnson, 2016), and so they may be understandably wary of study clinicians. Furthermore, people of color generally like to get to know people before opening up, which can make it difficult to elicit important trauma details at first contact. Moreover, people of color generally have a lower mental health literacy, due to greater cultural stigmas about mental health; therefore, study screening staff may need to provide psychoeducation about symptoms to aid in determining eligibility (Cheng, Wang, McDermott, Kridel, \& Rislin, 2018; Williams, Domanico, Marques, Leblanc, \& Turkheimer, 2012).

At the UConn site, we deliberately dedicated more time to talking to potential participants during phone screenings. Although this task was often performed by the study coordinator at other sites, we shifted to having the potential therapy providers make some of these calls to help them build rapport sooner. Therapists spent more time explaining PTSD symptoms to potential participants, or in some cases translating their descriptions of symptoms into terminology more consistent with DSM-5. Finally, therapists used this opportunity to collect or confirm details about the person's racial and ethnic identity to determine if the individual's participation would help meet our site's critical diversity goals.

\section{Informed consent}

The consent form is vitally important as it is a formal description of what the study entails. However, sometimes the document is misunderstood due to language that can be unnecessarily off-putting or frightening. As such, a change of language in consent forms was needed to make them more palatable for people of color. For example, use of the term "research" was minimized in favor of less triggering terms such as "project" and "study" to distance these recruitment efforts from associations with historical research abuses against African Americans (Williams, Tellawi, et al., 2013). For Latinx people, the word "investigation" can be triggering to those who face immigration stigma, so that was similarly reworded. "Experimental session" was changed to "overnight test session" to eliminate terms that may make participants further question their safety.

Researchers should also anticipate that more time will be needed to review paperwork with participants. They should make the process as conversational as possible to help put participants at ease. Because the informed consent document may resemble a contract, it is important to emphasize that participants are not signing away their rights, but rather the consent form exists to ensure that clinicians are doing their jobs by providing complete information about what to expect; participants are still free to withdraw at any time. As researchers begin to create a shared language with participants, space opens for participants to ask more questions about psychedelics, and this is a good opportunity to provide this education about psychedelics and debunk any myths they may believe.

It is important to review basic safety information, as that will be one of the participant's primary concerns. Participants of color are already wary for a number of reasons previously discussed and do not want to hear extreme details on everything that can go wrong - they are already worried about this and have decided to move forward anyway. Their main concerns about MDMA are fears of death, physical disability, mental disability, and addiction (Rigg \& Lawental, 2018), and so clinicians should focus on the track record to date for MDMA in these areas. Here, it would be important to give honest information - that no one who has taken MDMA in similar studies has died, become disabled, or become addicted (e.g., the MDMA Investigator's Brochure; Vizeli \& Liechti, 2017). Giving exact numbers helps promote trust and puts risks into perspective.

\section{Setting and music}

Set and setting refer to the non-drug factors, like mindset and physical surroundings that shape psychedelic experiences. People across cultures have been mindful of these factors in psychedelic healing practices for a very long time (Neitzke-Spruill, this issue). Therefore, it is critical to take stock of the messages implicit in the setting provided for participants. Individuals are part of a social context, which refers to the immediate physical and social setting in which people live or in which something happens or develops. It includes the person's culture and the people and institutions with whom they interact. Given the importance of social context, there are many facets to be considered when creating a comfortable space, which differs by culture. Because of the deeply entrenched and pervasive racist messages embedded in nearly every social structure, deliberate measures may be needed to create a welcoming venue. For example, a lack of diversity in artwork, reading material, and decorations may communicate threat to people of color (e.g., Purdie-Vaughns, Steele, Davies, Ditlmann, \& Crosby, 2008). When creating ambiance, clinicians should consider the décor of the facility - color schemes, choice of magazines, and cultural office artwork can all be critically important in making clients feel safe and welcome (Williams, BeckmannMendez, et al., 2013).

A great deal of care and forethought went into the décor of our study room at UConn, and input was solicited from all members of the team. In terms of artwork, on one wall hangs a framed print of a Latina mother holding her baby and on another an impressionist map of the world in multicolor. Other decorations include jade beads, a Tibetan sound bowl, and colorful cotton throws and pillows with patterns from India. As these decorations were reflective of the varied cultures of the team, they brought an element of cultural authenticity to the milieu. However, as noted, setting encompasses more than simply décor.

Since before the advent of Western psychedelic therapies, music has played an important role within the 
therapeutic framework as a way to provide support throughout the session (Bonny \& Pahnke, 1972). There is growing research to indicate that music can help facilitate psychedelic experiences that are beneficial and important therapeutically, and that listening to music while taking a psychedelic medicine can evoke and intensify emotions such as wonder, transcendence, power, tenderness, peacefulness, nostalgia, and joy (e.g., Kaelen, Roseman, Kahan, Santos-Ribeiro, \& Orban, 2016). In addition, music and song are the integral part of many indigenous psychedelic traditions (e.g., the icaros sung by shamanic healers during the traditional ayahuasca ceremony; Labate \& Pacheco, 2010; Tafur, 2017). In one study of 19 patients undergoing psychedelic therapy with psilocybin for depression, analyses revealed that the music had both "welcome" (desirable) and "unwelcome" (aversive) influences on the patient's experience (Kaelen, Giribaldi, Raine, \& Evans, 2018). In many cases, it evoked deeply meaningful, emotional, and therapeutically valuable emotions and imagery. In some cases, however, this was not the case; 6 out of 19 patients referred to "misguidance" and 9 out of 19 spoke to "resistance" as being a part of their experience at some point (Kaelen et al., 2018). However, to date, no empirical research has been conducted to examine the role of music in psychedelic-assisted therapy with people of color.

Music is strongly connected to culture, and it makes sense that music conducive to psychedelic healing for White Americans may be inadequate for those from other cultural traditions. Therefore, it is important to consider potential alterations, supportive measures, or alternatives to the typical Western playlist (e.g., new age music, space music, folk, post rock, etc.) that will better suit the individual needs of each patient based on their preferences, safety needs, age, and rich cultural background and heritage. At our site, we carefully tailored the playlist to the relevant demographic and cultural factors of our participants to help optimize their experience. As psychedelic therapy expands from research labs and into more clinics, we hope that more therapists will explore novel ways of incorporating music into psychedelic therapies with the diversity of individuals and cultural groups in mind.

\section{Training for team members}

It is of the utmost importance that study personnel have a genuine commitment to the mental health of people of color and have been properly trained in culturally informed assessment and treatment techniques. Although most new clinicians are now receiving some sort of diversity education in training programs to increase awareness, practical skills may not have been included. In addition, previous generations of clinicians may have received no diversity training at all, leaving them ill equipped to work with people of color.

At UConn, therapists were trained to take a multicultural approach to treatment, whereby the differences, strengths, and uniqueness of each ethnoracial group were embraced. All therapists participated in an 8-week diversity training where they learned to better understand and connect with people of color using Functional Analytic Psychotherapy (FAP; Tsai et al., 2009). Although FAP can be used as a standalone treatment for relationship issues, it is typically used in combination with other therapies to help increase engagement and strengthen rapport. The FAP training provided an experiential approach for recognizing and combating personal biases, and therapists learned how to respond to racially charged material in an empathetic rather than defensive or dismissive manner (Miller, Williams, Wetterneck, Kanter, \& Tsai, 2015). Therapists were also educated on microaggressions, including how to recognize and manage them in interactions. These trainings did not replace the nondirective, inner focused therapy that was required as per the MAPS PBC study protocol (Mithoefer, 2015), but rather augmented that approach.

Through a justice-oriented framework, our therapists provided an environment of openness, curiosity, and validation for our participants' racialized experiences. This included expressing empathy for experiences of racism and marginalization with verbal and non-verbal cues. It also included validating any mistrust of the research process, and acknowledgment of historical research abuses against people of color. All therapists were well aware of structural racism typically embedded in current research processes and vocalized their commitment to doing things differently. As part of the conversation during initial visits, our therapists might say something like, "One reason this site is committed to working with people of color is because almost all research to date has been done with White people. They have been getting this treatment for years, and it's time that people like us are included too. Otherwise, how do we even know if this treatment makes sense for us and our communities? Your involvement will help move this work forward for everyone, and in the process you will receive a culturallyinformed treatment experience."

Finally, cultural humility was emphasized throughout. Despite the diversity of our team, we could not be equipped for every ethnic and cultural group that contacted us, so we needed to be sure not to ever become complacent in our efforts or stop learning. Cultural humility is an approach that communicates attentiveness and interest in the other person, marked by openness, curiosity, lack of arrogance, and genuine desire to understand clients' cultural identities (Mosher et al., 2017). Therapists with high levels of cultural humility rarely assume competence, but rather approach clients with respectful openness and collaboratively explore the client's cultural identities. In this regard, cultural competence should be viewed as a journey, not a destination, as we are always becoming more culturally competent while never actually achieving cultural competence. Cultural competence is a dynamic ever-changing and ever-challenging lifelong process (Yeager \& Bauer-Wu, 2013).

\section{Assessing racial trauma}

When traumatization results from experiences of racism, it is sometimes called racial trauma. It can be caused by major experiences of racism such as workplace harassment or police brutality, or it can be the result of an accumulation of many small occurrences, such as everyday discrimination and microaggressions (e.g., Williams, Kanter, \& Ching, 2018). One difficulty is that clinicians may only recognize racism as trauma when an individual experiences a specific racist event, such as a violent hate crime 
(Malcoun, Williams, \& Bahojb-Nouri, 2015). This is limiting given that many people of color experience cumulative experiences of racism as traumatic (Butts, 2002). In addition, people of color may not share experiences of discrimination when asked about traumas they may have experienced unless they are told explicitly that racism counts as a trauma (Williams, Metzger, Leins, \& DeLapp, 2018).

To ensure that traumas due racism and to other forms of oppression were captured, a modified version of the UConn Racial/Ethnic Stress \& Trauma Survey (UnRESTS; Williams, Metzger, et al., 2018) was added to the source documents used for collecting information about participants by study therapists. The UnRESTS is a semi-structured interview designed to assess racial trauma in clients who are part of stigmatized ethnic and racial groups. The UnRESTS collects information about a client's ethnic identity and then guides the clinician to ask about experiences surrounding: (a) explicit and obvious racism, (b) discrimination experienced by loved ones, (c) vicarious prejudicial experiences that were learned about, and (d) experiences with subtle forms of racism or microaggressions. The scope of the measure was broadened to include other types of discrimination, including "gender, sexual orientation, age, disability, faith, or a combination of reasons," and it was shortened from its original length to minimize participant burden. This information is also provided to the independent raters so they know about the participants' experiences as well. Feedback from clinicians at various MAPS PBC study sites found the measure easy to use and useful in better understanding their participants' histories of racial trauma and other forms stigmatization. Even in cases where experiences of discrimination did not meet DSM-5 Criterion A, the information was useful for better understanding participants' lived experiences (see "Supplementary Material" for the short version of the UnRESTS).

\section{Training for independent raters}

The independent raters for the MDMA-assisted psychotherapy for PTSD study are gatekeepers in a sense, as they quantify the PTSD diagnosis for participants, which must meet a certain threshold before being allowed to enroll. Using the gold standard in PTSD research, the Clinicians Administered PTSD Scale (Weathers et al., 2013), the independent rater group is a pool of highly trained clinicians who are not present during therapy to minimize bias during outcome measurements.

As with study therapy providers, participants of color may not initially trust raters, thereby requiring raters to develop a quick positive rapport with participants, so they feel safe enough to disclose their traumas. Furthermore, raters needed to be able to identify sources of trauma related to the experience of racial discrimination and related forms of oppression. However, the vast majority of MAPS PBC independent raters were White and many had inadequate training in working with people of color. Since the study design required that participants receive their rater from the pool randomly, it was not possible to provide an ethnic match for participants of color or even make a rater recommendation to ensure that the clinician was well-trained in working with people of color.
To address these issues, more raters of color were hired and all raters received a diversity training designed in part by the first author (MTW) to better assess clients across racial, ethnic, and cultural differences. This included regular meetings online as a group where raters were provided readings and presentations on the topics of ethnic identity development, therapeutic distance based on ethnic identity; models of acculturation; Whiteness and privilege; racism and microaggressions; racial trauma, personality disorders, and culture; and cultural issues surrounding the use of study measures. These trainings were video-recorded and are now required viewing for MAPS PBC therapy providers participating in Phase 3 studies, which extends the benefits of this training study-wide. In addition, two short quizzes were developed by the third author (RA) that allowed therapy providers to reflect on the material presented.

Independent raters also completed challenging experiential homework exercises designed to facilitate a shift in thinking from an ethnocentric to a multicultural perspective. This involved practicing mutually reciprocal interactions with others across race, practicing giving the UnRESTS, self-reflection, and sharing challenges in the group. Similar techniques have been empirically shown to improve clientclinician interactions (Rosen et al., 2018). The raters learned much from this training and felt better equipped to work with diverse participants (see "Supplementary Materials" for questions used to help assess rater cultural competency). Even so, this situation was not perfect - a Caribbean woman who was selected to be a part of the trial dropped out of screening because she did not receive a Black woman as her rater.

\section{Institutional resistance}

Despite initial displays of enthusiasm about the study by university leadership, there was some fear and misunderstanding surrounding safety issues related to MDMA. This led to unreasonable levels of precaution, far greater than warranted based on MDMA's safety record and exceeding what was required by the FDA. Although the study was designed and approved for an outpatient setting, the institution's Office of the Vice President of Research (OVPR) insisted the study to take place in the hospital, which added greatly to the level of bureaucracy, complexity of study billing, and study costs (Lekhtman, 2018). On several occasions, the health center demanded the employment of a nurse as a night attendant instead of a volunteer, as is typically done at other study sites. We opted to use a clinical graduate student as a night attendant (with the study physician and PI on call), as a nurse was unnecessary and would have been cost prohibitive.

There was notable structural, institutional, and individual racism that also impeded the work, as well as bureaucratic obstacles. This included untenable delays in hiring essential personnel, including 5 months to officially hire the study coordinator. The PI had to meet with the OVRP and the Chief Diversity Officer to overcome this resistance, and once hired, the institution refused to provide the study coordinator with her back pay, even though the sponsor had already provided these funds to the university. We were able to resolve this by having the sponsor pay the study 
coordinator to back pay directly. Other barriers included refusal to provide promised billing services, refusal to give prescribing privileges to our team's psychiatrist (a Harvardtrained MD-PhD), twice having our study space given away to another research team, study staff being harassed by nurses for "not belonging" in our research space, an aggressive audit of the study that took 2 months of staff time (and included several inaccurate findings of misconduct that were later withdrawn), opposing the PI's plans to move the study to a more collegial health center division, and inappropriate threats by the OVPR for exploring the possibility of moving the study off-campus.

In terms of sexism, female clinicians felt ignored and not taken seriously by health center staff and UConn leadership. For example, there were several meetings by chairs, deans, and vice presidents about study issues and in every case the female PI (MTW) was not invited, despite repeated requests for inclusion; rather she was informed about decisions impacting the study after the fact, without the benefit of her knowledge and input at those meetings.

There were other problematic issues as well, such as higher than normal charges by the health center for medical procedures, in some cases three times the typical rate (e.g., the health center charged the sponsor $\$ 3,820$ for a nuclear stress test while charging Anthem only $\$ 837$ for this same test). In this case, we were able to contract with a less expensive outside clinic for needed cardiac tests.

\section{DISCUSSION}

\section{Implications for Phase 3 trials}

In this study, we have outlined several key issues and strategies surrounding participation and retention of people of color into MDMA-assisted psychotherapy studies. Important considerations include a diverse study staff, purposeful training in cultural competence for therapists and raters, targeted outreach for recruitment, meaningful incentives, a comfortable setting, and competent assessment of racial trauma. Critical discussion on limitations and obstacles to recruiting diverse populations is important to conduct during protocol development. It is worth noting that involving someone with expertise in culturally informed research methods at the front end, before protocols were developed, would have prevented some of the methodological issues that needed to be changed after the fact. There is also a need to confront institutional and individual biases that may work against inclusion, as racism and sexism are embedded into nearly all institutional structures and systems (Salter, Adams, \& Perez, 2018). For example, currently, MAPS PBC staff includes only 4 people of color out of 26. Recognizing the benefits of a diverse staff, the organization is in the process of hiring with the intention of increasing diversity, particularly in the upper echelons of the organization.

The methods described herein were effective for recruitment and retention at our site in Connecticut but each community has its own differences and nuances, which will require an ongoing review of efforts to determine if diversity goals are being met. Holding regular team meetings to review enrollment rates versus targeted enrollment numbers can inform team leaders as to if diversity goals are being met. A self-correcting process should be implemented whereby methods that are not effective are reduced and those that are effective are retained or increased (Clay, Ellis, Amodeo, Fassler, \& Griffin, 2003).

MAPS PBC is currently running Phase 3 trials, and although the UConn site was not included due to lack of adequate institutional support at the health center (Lekhtman, 2018), many of the modifications made to study protocols continue to benefit other sites and the study as a whole, such as the changes to consent forms, trainings for raters, and training materials for therapy providers. However, people of color are underrepresented as clinicians at most sites, so work is still needed to make participants of color feel included, welcome, safe, and understood. As a result, there may be less than representative proportions of participants from various ethnoracial groups in the final sample. If the final Phase 3 sample does not contain enough patients of color for meaningful subgroup analyses, an additional study focused on people of color would be an important next step to help determine the safety and efficacy of MDMA-assisted psychotherapy for people in underrepresented racial and ethnic groups.

\section{Expanded Access}

Expanded Access is an FDA program that allows the use of an investigational drug under an approved treatment protocol. The program is designed to address urgent and lifethreatening conditions in patients who do not adequately respond to available treatment options. MAPS PBC has recently applied for the Expanded Access program and hopes for approval in 2019. If Expanded Access is approved, new sites in the US will be able to participate in their Expanded Access protocol, which would allow the provision of open-label MDMA-assisted psychotherapy for PTSD. Qualified sites will be required to have a team of therapy providers, a physician, a study coordinator, and a facility suitable to conduct MDMA-assisted psychotherapy, with approval by regulatory agencies and under supervision of MAPS PBC. Furthermore, therapy providers must be trained through the MAPS Therapy Training Program to be eligible to provide therapy, and this training and supervision process costs approximately $\$ 9,000$ per therapist. Expanded Access clinics can utilize the methods described here to increase inclusion of patients of color. That being said, due to the extensive site and provider requirements, the cost for an entire course of treatment will be unaffordable for many people (current estimates are approximately $\$ 15,000$ ), and so work is needed to make this treatment accessible to those with limited financial means.

As of 2018, MAPS PBC trained 221 MDMA therapists, and only $22(<10 \%)$ of these are therapy providers of color, which included international therapy providers. More therapists of color are sorely needed to bring MDMA treatment to communities of color during the Expanded Access period and thereafter. To this end, MAPS PBC is co-sponsoring an MDMA therapy training for communities of color, grantfunded by Open Society Foundations, Riverstyx Foundation, Libra Foundation, and others sponsors. This training 
will equip therapists and inform community leaders of color about this new modality and its ability to reduce symptoms of PTSD, with a focus on culturally informed psychedelic therapy. In the process of developing this training, the standard MAPS PBC trainings will also benefit, as many culturally informed updates will be available to add into it. In addition to the standard 6.5-day training, this special training will feature a 1.5-day opening workshop entitled, "Psychedelic Medicine and Cultural Trauma." Community leaders will join therapy providers to engage in dialogue about the social, political, cultural, and historical causes of trauma, as well as a history of indigenous practices of healing with plant medicines. This workshop will discuss barriers to acceptance of MDMA therapy in communities of color and potential ways forward. Most importantly, this training will help build a community of color dedicated to bringing MDMA therapy to communities to support its members to both flourish and expand.

\section{Future psychedelic therapists}

Leaders in the field of psychedelic medicine are working to develop credentialing boards to implement the therapeutic competencies needed to work responsibly with MDMA and other psychedelic substances. Because of the importance of this work and the extreme vulnerability of patients during non-ordinary states of consciousness, all therapy providers and supervisors should have basic competencies in working ethically and skillfully with people of color (Phelps, 2017; Sisti, 2018). These essential skills include the ability to (a) understand normal cultural variations in the expression of psychopathology and personality traits; (b) identify trauma related to the experience of racial discrimination, ethnic violence, xenophobia, and other forms of oppression; (c) develop good rapport with people of color by appropriately expressing caring, empathy, and respect; (d) comfortably engage in discussions about racially charged topics; and (e) identify and describe their own biases as they relate to ethnic and racial differences, with an ongoing action plan to address these. All therapy providers conducting psychedelic therapies should be trained and tested in these areas prior to certification (also see "Supplementary materials" for assessing cultural competency).

\section{CONCLUSIONS}

A culturally informed approach in psychedelic therapies can and should be used in the research process to ensure equity and diversity in access to the potential benefits of MDMA. Current processes for recruitment and treatment are not sufficient to open the doors of psychedelic therapies to people of color in the US or internationally (Michaels et al., 2018). MAPS has been a leader in conducting research into MDMA-assisted therapy and to our knowledge has been the first to make a deliberate effort to include of people of color in the work. Still more work is needed and hopefully this trajectory of inclusion will continue as other groups begin the work of ensuring that psychedelic research meets the needs of everyone.
Acknowledgements: The authors would like to thank Multidisciplinary Association for Psychedelic Studies (MAPS) for support of the study "An Open-Label, Multi-Site Phase 2 Study of the Safety and Effect of Manualized MDMAAssisted Psychotherapy for the Treatment of Severe Posttraumatic Stress Disorder (MP-16)." They would also acknowledge the Open Society Foundations (OSF), Libra Foundation, Riverstyx Foundation, and others for grant funding for "MDMA Therapy Training for Communities of Color." In addition, they would acknowledge Chad Wetterneck for providing diversity training and materials for the MAPS rater pool, Alli Feduccia for her comments on the manuscript, and Jennifer Purdon for help with the literature search.

Conflict of interest: All authors report no conflicts of interest.

\section{REFERENCES}

Avery, D. R., Hernandez, M., \& Hebl, M. R. (2004). Who's watching the race? Racial salience in recruitment advertising. Journal of Applied Social Psychology, 34(1), 146-161. doi:10.1111/j.1559-1816.2004.tb02541.x

Bonny, H. L., \& Pahnke, W. N. (1972). The use of music in psychedelic (LSD) psychotherapy. Journal of Music Therapy, 9(2), 64-87. doi:10.1093/jmt/9.2.64

Bosson, R., Williams, M. T., Lippman, S., Carrico, R., Kanter, J., Peña, A., Mier-Chairez, J., \& Ramirez, J. (2017). Addressing refugee mental health needs: From concept to implementation. The Behavior Therapist, 40(3), 110-112.

Breslau, N., Chilcoat, H. D., Kessler, R. C., \& Davis, G. C. (1999). Previous exposure to trauma and PTSD effects of subsequent trauma: Results from the Detroit Area Survey of Trauma. American Journal of Psychiatry, 156(6), 902-907. doi:10.1176/ajp.156.6.902

Burns, J. (2017, August 28). FDA designates MDMA as 'Breakthrough Therapy' for post-traumatic stress. Forbes. Retrieved from https://www.forbes.com/sites/janetwburns/2017/ 08/28/fda-designates-mdma-as-breakthrough-therapy-for-posttraumatic-stress/\#2ebcd1f27460

Butts, H. F. (2002). The black mask of humanity: Racial/ ethnic discrimination and post-traumatic stress disorder. The Journal of the American Academy of Psychiatry and the Law, 30, 336-339.

Cheng, H., Wang, C., McDermott, R. C., Kridel, M., \& Rislin, J. L. (2018). Self-stigma, mental health literacy, and attitudes toward seeking psychological help. Journal of Counseling \& Development, 96(1), 64-74. doi:10.1002/jcad.12178

Ching, T. H. W. (in press). Intersectional insights from an MDMAassisted psychotherapy training trial: An open letter to racial/ ethnic and sexual/gender minorities. Journal of Psychedelic Studies.

Clay, C., Ellis, M. A., Amodeo, M., Fassler, I., \& Griffin, M. L. (2003). Families in society: Recruiting a community sample of African American subjects: The nuts and bolts of a successful effort. Families in Society, 84(3), 396-404. doi:10.1606/10443894.111 
Emerson, A., Ponté, L., Jerome, L., \& Doblin, R. (2014). History and future of the Multidisciplinary Association for Psychedelic Studies (MAPS). Journal of Psychoactive Drugs, 46(1), 27-36. doi:10.1080/02791072.2014.877321

Fisher, C. B., Hoagwood, K., Boyce, C., Duster, T., Frank, D. A., Grisso, T., Levine, R. J., Macklin, R., Spencer, M. B., Takanishi, R., Trimble, J. E., \& Zayas, L. H. (2002). Research ethics for mental health science involving ethnic minority children and youth. American Psychologist, 57, 1024-1039.

Grady, C. (2005). Payment of clinical research subjects. The Journal of Clinical Investigation, 115(7), 1681-1687. doi:10.1172/JCI25694

Herzberg, G., \& Butler, J. (2019, March 13). Blinded by the White: Addressing power and privilege in psychedelic medicine. Chacruna. Retrieved from https://chacruna.net/blindedby-the-white-addressing-power-and-privilege-in-psychedelicmedicine

Himle, J. A., Baser, R. E., Taylor, R. J., Campbell, R. D., \& Jackson, J. S. (2009). Anxiety disorders among African Americans, Blacks of Caribbean descent, and non-Hispanic Whites in the United States. Journal of Anxiety Disorders, 23(5), 578-590. doi:10.1016/j.janxdis.2009.01.002

Kaelen, M., Giribaldi, B., Raine, J., \& Evans, L. (2018). The hidden therapist: Evidence for a central role of music in psychedelic therapy. Psychopharmacology, 235(2), 505-519. doi:10.1007/s00213-017-4820-5

Kaelen, M., Roseman, L., Kahan, J., Santos-Ribeiro, A., \& Orban, C. (2016). LSD modulates music-induced imagery via changes in parahippocampal connectivity. European Neuropsychopharmacology, 26(7), 1099-1109. doi:10.1016/j.euroneuro. 2016.03.018

Kessler, R. C., Aguilar-Gaxiola, S., Alonso, J., Benjet, C., Bromet, E. J., Cardoso, G., Degenhardt, L., de Girolamo, G., Dinolova, R. V., Ferry, F., Florescu, S., Gureje, O., Haro, J. M., Huang, Y., Karam, E. G., Kawakami, N., Lee, S., Lepine, J. P., Levinson, D., Navarro-Mateu, F., Pennell, B. E., Piazza, M., Posada-Villa, J., Scott, K. M., Stein, D. J., Ten Have, M., Torres, Y., Viana, M. C., Petukhova, M. V., Sampson, N. A., Zaslavsky, A. M., \& Koenen, K. C. (2017). Trauma and PTSD in the WHO World Mental Health Surveys. European Journal of Psychotraumatology, 8(Suppl. 5), 1353383. doi:10.1080/ 20008198.2017.1353383

Labate, B. C., \& Pacheco, G. (2010). Opening the portals of heaven: Brazilian Ayahuasca music. Munich, Germany: Lit Verlag.

Lekhtman, A. (2018, December 18). Pioneering UConn MDMA research focused on people of color ends early: What are the next steps for equity in treatment? Psychedelic Times. Retrieved from https://psychedelictimes.com/2018/12/18/ pioneering-uconn-mdma-research-focused-on-people-of-colorends-early-what-are-the-next-steps-for-equity-in-treatment/

Maina, I. W., Belton, T. D., Ginzberg, S., Singh, A., \& Johnson, T. J. (2016). A decade of studying implicit racial/ethnic bias in healthcare providers using the implicit association test. Social Science and Medicine, 199, 219-229. doi:10.1016/ j.socscimed.2017.05.009

Malcoun, E., Williams, M. T., \& Bahojb-Nouri, L. V. (2015). Assessment of Posttraumatic Stress Disorder in African Americans. In L. T. Benuto \& B. D. Leany (Eds.), Guide to psychological assessment with African Americans (pp. 163-182). New York, NY: Springer.
Michaels, T. I., Purdon, J., Collins, A., \& Williams, M. T. (2018). Inclusion of people of color in psychedelic-assisted psychotherapy: A review of the literature. BMC Psychiatry, 18(245), 1-9. doi:10.1186/s12888-018-1824-6

Miller, A., Williams, M. T., Wetterneck, C. T., Kanter, J., \& Tsai, M. (2015). Using functional analytic psychotherapy to improve awareness and connection in racially diverse client-therapist dyads. The Behavior Therapist, 38(6), 150-156.

Mithoefer, M. C. (2015, August). A manual for MDMA-assisted psychotherapy in the treatment of posttraumatic stress disorder, Version 7: 19. Santa Cruz, CA: Multidisciplinary Association for Psychedelic Studies (MAPS). Retrieved from https://maps. org/research-archive/mdma/MDMA-Assisted-PsychotherapyTreatment-Manual-Version7-19Aug15-FINAL.pdf

Mithoefer, M. C., Mithoefer, A. T., Feduccia, A. A., Jerome, L., Wagner, M., Wymer, J., Holland, J. T., Hamilton, S., YazarKlosinski, B., Emerson, A. B., \& Doblin, R. (2018). 3, 4-Methylenedioxymethamphetamine (MDMA)-assisted psychotherapy for post-traumatic stress disorder in military veterans, firefighters, and police officers: A randomised, double-blind, dose-response, phase 2 clinical trial. The Lancet Psychiatry, 5(6), 486-497. doi:10.1016/S2215-0366(18)30135-4

Mithoefer, M. C., Wagner, M. T., Mithoefer, A. T., Jerome, L., \& Doblin, R. (2011). The safety and efficacy of \pm 3 , 4-methylenedioxymethamphetamine-assisted psychotherapy in subjects with chronic, treatment-resistant posttraumatic stress disorder: The first randomized controlled pilot study. Journal of Psychopharmacology, 25(4), 439-452. doi:10.1177/ 0269881110378371

Mosher, D. K., Hook, J. N., Captari, L. E., Davis, D. E., DeBlaere, C., \& Owen, J. (2017). Cultural humility: A therapeutic framework for engaging diverse clients. Practice Innovations, 2(4), 221-233. doi:10.1037/pri0000055

Phelps, J. (2017). Developing guidelines and competencies for the training of psychedelic therapists. Journal of Humanistic Psychology, 57(5), 450-487. doi:10.1177/0022167817711304

Pole, N., Gone, J. P., \& Kulkarni, M. (2008). Posttraumatic stress disorder among ethnoracial minorities in the United States. Clinical Psychology: Science and Practice, 15(1), 35-61. doi:10.1111/j.1468-2850.2008.00109.x

Purdie-Vaughns, V., Steele, C. M., Davies, P. G., Ditlmann, R., \& Crosby, J. R. (2008). Social identity contingencies: How diversity cues signal threat or safety for African Americans in mainstream institutions. Journal of Personality and Social Psychology, 94(4), 615-630. doi:10.1037/0022-3514.94.4.615

Rigg, K. K., \& Lawental, M. (2018). Perceived risk associated with MDMA (Ecstasy/Molly) use among African Americans: What prevention and treatment providers should know. Substance Use \& Misuse, 53(7), 1076-1083. doi:10.1080/10826084. 2017.1392985

Rosen, D. C., Kanter, J. W., Manbeck, K., Branstetter, H., Corey, M. D., Williams, M. T., \& Masuda, A. (2018, July). A contextual-behavioral intervention to improve provider empathy and emotional rapport in racially charged interactions: A randomized trial. In M. D. Corey (Chair), Applying CBS to address social divisions. Symposium presentation at ACBS World Conference 16, Montréal, Québec.

Salter, P. S., Adams, G., \& Perez, M. J. (2018). Racism in the structure of everyday worlds: A cultural-psychological perspective. Current Directions in Psychological Science, 27(3) 150-155. doi:10.1177/0963721417724239 
Sisti, D. (2018). Creating an ethical framework for psychedelic therapy research. Multidisciplinary Association for Psychedelic Studies (MAPS) Bulletin, 28(3), 34-35.

Suite, D. H., La Bril, R., Primm, A., \& Harrison-Ross, P. (2007). Beyond misdiagnosis, misunderstanding and mistrust: Relevance of the historical perspective in the medical and mental health treatment of people of color. Journal of the National Medical Association, 99(8), 879-885.

Tafur, J. (2017). Fellowship of the river. Pheonix, AZ: Espirito Books.

Tsai, M., Kohlenberg, R. J., Kanter, J. W., Kohlenberg, B., Follette, W. C., \& Callaghan, G. M. (2009). A guide to functional analytic psychotherapy: Awareness, courage, love, and behaviorism. New York, NY: Springer.

U.S. Department of Health and Human Services. (1999). The fundamentals of mental health and mental illness, Ch 2. In Mental health: A report of the surgeon general. Rockville, MD: U.S. Department of Health and Human Services, Substance Abuse and Mental Health Services Administration, Center for Mental Health Services, National Institutes of Health, National Institute of Mental Health.

Vizeli, P., \& Liechti, M. E. (2017). Safety pharmacology of acute MDMA administration in healthy subjects. Journal of Psychopharmacology, 31(5), 576-588. doi:10.1177/0269881117691569

Weathers, F. W., Blake, D. D., Schnurr, P. P., Kaloupek, D. G., Marx, B. P., \& Keane, T. M. (2013). The clinicianadministered PTSD scale for DSM-5 (CAPS-5). Interview retrievedfrom the National Center for PTSD at www.ptsd.va.gov

Williams, M. T., Beckmann-Mendez, D., \& Turkheimer, E. (2013). African American attitudes about participation in anxiety disorders research. Journal of the National Medical Association, 105(1), 33-41. doi:10.1016/S0027-9684(15)30083-3
Williams, M. T., Domanico, J., Marques, L., Leblanc, N., \& Turkheimer, E. (2012). Barriers to treatment among African Americans with obsessive-compulsive disorder. Journal of Anxiety Disorders, 26(4), 555-563. doi:10.1016/j.janxdis. 2012.02.009

Williams, M. T., Kanter, J. W., \& Ching, T. H. W. (2018). Anxiety, stress, and trauma symptoms in African Americans: Negative affectivity does not explain the relationship between microaggressions and psychopathology. Journal of Racial and Ethnic Health Disparities, 5(5), 919-927. doi:10.1007/ s40615-017-0440-3

Williams, M. T., \& Leins, C. (2016). Race-based trauma: The challenge and promise of MDMA-assisted psychotherapy. Multidisciplinary Association for Psychedelic Studies (MAPS) Bulletin, 26(1), 32-37.

Williams, M. T., Metzger, I., Leins, C., \& DeLapp, C. (2018). Assessing racial trauma within a DSM-5 framework: The UConn Racial/Ethnic Stress \& Trauma Survey. Practice Innovations, 3(4), 242-260. doi:10.1037/pri0000076

Williams, M. T., Printz, D., Ching, T., \& Wetterneck, C. T. (2018). Assessing PTSD in ethnic and racial minorities: Trauma and racial trauma. Directions in Psychiatry, 38(3), 179-196.

Williams, M. T., Proetto, D., Casiano, D., \& Franklin, M. E. (2012). Recruitment of a hidden population: African Americans with obsessive-compulsive disorder. Contemporary Clinical Trials, 33(1), 67-75. doi:10.1016/j.cct.2011.09.001

Williams, M. T., Tellawi, G., Wetterneck, C. T., \& Chapman, L. K. (2013). Recruitment of ethnoracial minorities for mental health research. The Behavior Therapist, 36(6), 151-156.

Yeager, K. A., \& Bauer-Wu, S. (2013). Cultural humility: Essential foundation for clinical researchers. Applied Nursing Research, 26(4), 251-256. doi:10.1016/j.apnr.2013.06.008 